\title{
Randomized Controlled Trial for Efficacy of Capsular Distension for Adhesive Capsulitis: Fluoroscopy-Guided Anterior Versus Ultrasonography-Guided Posterolateral Approach
}

\author{
Jae Hyun Bae, $\mathrm{MD}^{1}$, Young Sook Park, $\mathrm{MD}^{1}$, Hyun Jung Chang, $\mathrm{MD}^{1}$, Min Jung Kim, $\mathrm{MD}^{1}$, \\ Kang Young Park, $\mathrm{MD}^{1}$, Seung Hwan Jin, $\mathrm{MD}^{1}$, Eun Hee Lee, $\mathrm{MD}^{2}$
}

Departments of ${ }^{1}$ Physical Medicine and Rehabilitation, ${ }^{2}$ Pathology, Samsung Changwon Hospital, Sungkyunkwan University School of Medicine, Changwon, Korea

\begin{abstract}
Objective To find the most effective procedure to treat adhesive capsulitis of the shoulder, we evaluated the clinical effects of an ultrasonographic-guided anterior approach capsular distension and a fluoroscopyguided posterolateral approach capsular distension. We expected the anterior approach to be better than the posterolateral approach because the rotator interval, a triangular anatomic area in the anterosuperior aspect of the shoulder, which is considered an important component of the pathology of adhesive capsulitis.

Methods Participants were randomly assigned to two groups: 27 patients in group A were injected by an anterior approach with $2 \%$ lidocaine $(5 \mathrm{~mL})$, contrast dye $(5 \mathrm{~mL})$, triamcinolone $(40 \mathrm{mg})$, and normal saline (9 $\mathrm{mL}$ ) under fluoroscopic guidance in the operating room. Twenty-seven patients in group B were injected using a posterolateral approach with $2 \%$ lidocaine $(5 \mathrm{~mL})$, triamcinolone $(40 \mathrm{mg})$, and normal saline $(14 \mathrm{~mL}) \mathrm{under}$ ultrasonographic guidance. After injection, all patients received physiotherapy four times in the first postoperative week and then two times each week for eight more weeks. Treatment effects were assessed using the shoulder pain and disability index (SPADI), visual numeric scale (VNS), passive range of motion (PROM), hand power (grip and pinch) at baseline and at one week, five and nine weeks after injection.

Results SPADI, VNS, PROM, and hand power improved in one week, five and nine weeks in both groups. Statistically significant differences were not observed in SPADI, VNS, PROM, or hand power between groups.
\end{abstract}

Received June 10, 2013; Accepted October 17, 2013

Corresponding author: Young Sook Park

Department of Physical Medicine and Rehabilitation, Samsung Changwon Hospital, Sungkyunkwan University School of Medicine, 158 Paryong-ro, Masanhoewon-gu, changwon 630-723, Korea

Tel: +82-55-290-6390, Fax: +82-55-290-6588, E-mail: jijibaeheiwon@hanmail.net

(c) This is an open-access article distributed under the terms of the Creative Commons Attribution Non-Commercial License (http://creativecommons.org/ licenses/by-nc/3.0) which permits unrestricted noncommercial use, distribution, and reproduction in any medium, provided the original work is properly cited. Copyright $\odot 2014$ by Korean Academy of Rehabilitation Medicine 
Conclusion Ultrasonography-guided capsular distension by a posterolateral approach has similar effects to fluoroscopy-guided capsular distension by an anterior approach.

Keywords Adhesive capsulitis, Injection

\section{INTRODUCTION}

Adhesive capsulitis is characterized by spontaneous onset of shoulder pain especially at night and global limitation of both active and passive shoulder motion. In 1945, Neviaser and Neviaser [1] described the gross and histologic pathology in a series of cases and proposed the term 'adhesive capsulitis' as a more precise descriptor of the findings. Adhesive capsulitis is characterized by a thickened, tight glenohumeral joint capsule with adhesions obliterating the normally patulous axillary fold. The fibrotic capsule adheres to itself and to the anatomic neck of the humerus. Normal shoulder joint volumetric capacity is 28 to $35 \mathrm{~mL}$ of injected fluid, whereas in adhesive capsulitis, the joint accepts only 5 to $10 \mathrm{~mL}$ [2]. Biopsy of the capsule demonstrates a chronic inflammatory infiltrate, absence of a synovial lining, moderate to extensive subsynovial fibrosis, and perivascular lymphocytic reactions $[1,3]$. Treatment options documented in the literature include supervised physical rehabilitation, nonsteroidal anti-inflammatory medications, oral corticosteroids, intra-articular corticosteroid injection, ultrasound-guided pulsed-mode radiofrequency lesioning of the suprascapular nerve, botulinum toxin intra-articular injection, distension arthrography, closed manipulation, open surgical release, and arthroscopic capsular release [4-7]. Glenohumeral joint distension, which was first described in 1965, was performed at the time of surgery and repeated until visualization of capsular rupture [8]. With respect to improving range of motion, hydrodilation has been reported to be superior to manipulation performed in the anesthetized patient [9]. Treatment consisting of repeated hydrodilation plus steroid injection has been found to be more effective than a single hydrodilation in reducing disability and pain [10]. The capsular distension method with corticosteroid injection has a therapeutic effect, via rupture of the stiffened capsule by hydropressure and reduction of synovial inflammation and capsular fibrosis. This method allows for improvement of shoulder motion and pain with a deceased time to func- tional recovery [11-13].

Blind shoulder intra-articular injections, which are widely used in clinical settings, have reported accuracies ranging from $27 \%$ to $80 \%$, depending on approaches used. Ultrasonography allows for more precise needle positioning, is less time-consuming, and requires less radiation exposure than fluoroscopy [14].

Magnetic resonance imaging (MRI) has been used for the identification of changes in the appearance of the joint capsule and periarticular tissues (thickening, contrast enhancement, and obliteration of adjacent fat planes) at the rotator interval and joint capsule at the level of the axillary pouch with variable performances. Gondim Teixeira et al. [14] reported sensitivity and specificity values for the MRI signs (inferior glenohumeral ligament T2 hyperintensity, inferior glenohumeral ligament enhancement, subcoracoid fat triangle obliteration, coracohumeral ligament thickness, and rotator interval enhancement) of adhesive capsulitis. The highest specificity among these was $94.10 \%$ of rotator interval enhancement. The rotator interval is the triangular space located in the anterosuperior portion of the glenohumeral joint. It is bounded by the supraspinatus superiorly and the subscapularis inferiorly, and the coracoid process forms its medial base. Contained within this triangular space are the coracohumeral ligament, middle glenohumeral ligament, superior glenohumeral ligament, long head of the biceps tendon, and anterior joint capsule [15].

Harryman et al. [16] showed that sectioning of the rotator interval capsule and ligamentous structures increased passive glenohumeral flexion, extension, external rotation, and adduction in 80 shoulders of the first comprehensive cadaveric study. Therefore, we aimed to evaluate the clinical effect of fluoroscopy-guided capsular distension by an anterior approach compared with ultrasonography-guided capsular distension from a posterolateral approach. It was the hypothesis of this study that capsular distension by an anterior approach would have a better effect compared to capsular distension by a posterolateral approach. 


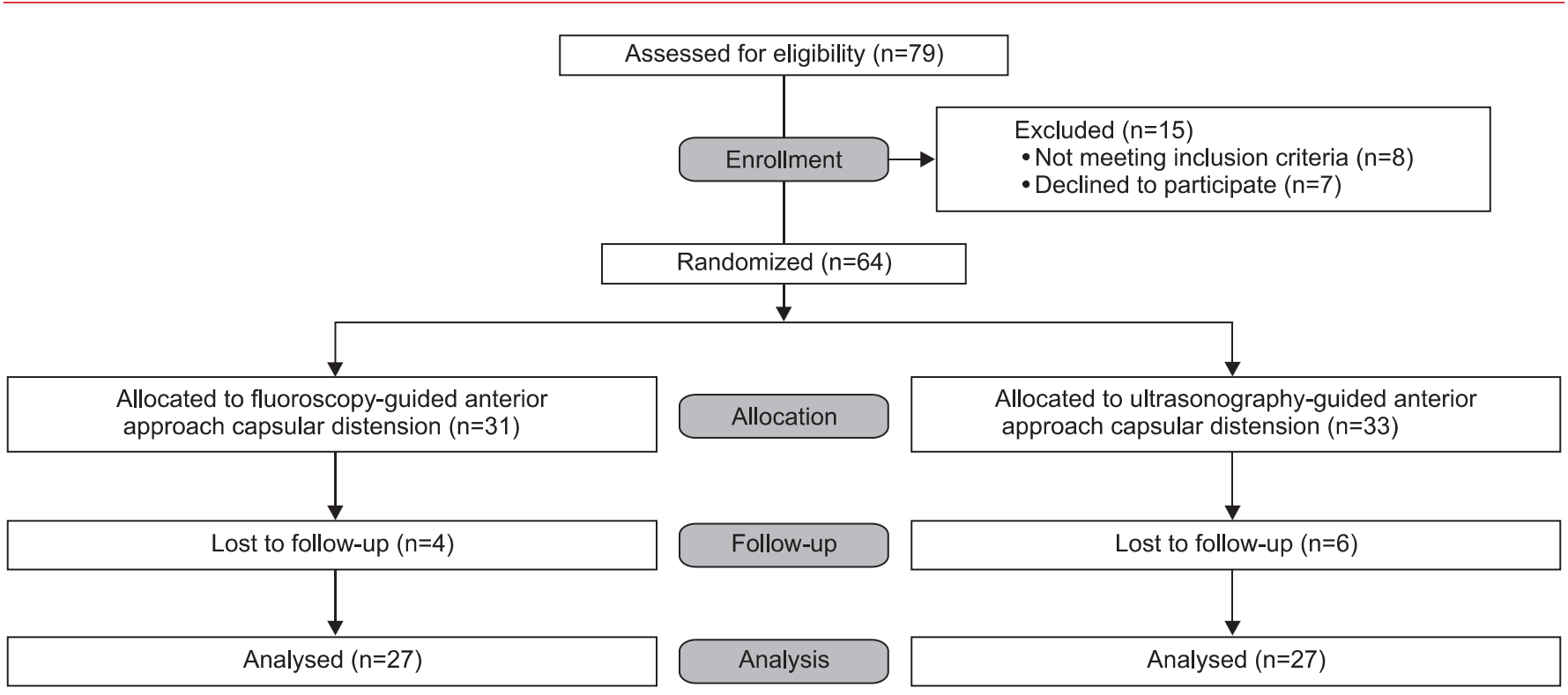

Fig. 1. Patient flowchart.

\section{MATARIALS AND METHODS}

\section{Participants}

From May 2011 to December 2012, 79 participants with shoulder pain and motion limitations who visited the Department of Rehabilitation Medicine of our hospital were enrolled. Fifteen participants were excluded because they did not meet criteria or declined to participate. Of the remaining 64 participants were assigned into one of two groups (group A, fluoroscopy-guided capsular distension with anterior approach; group B, ultrasonographyguided capsular distension with posterior approach). The allocation sequence was generated using a computer program (Microsoft Excel) simple randomization method. Except for 10 participants with follow-up loss, 54 participants were analyzed (Fig. 1). The exclusion criteria were shoulder surgery history and systemic disease that could affect shoulder arthritis. Even though included participants had received shoulder treatment for at least three months, they suffered from shoulder pain and global motion limitations, especially internal rotation and adduction direction. Age, gender, symptom duration, and symptom side were recorded.

\section{Methods}

Fluoroscopy-guided capsular distension method (group A)

The procedure was performed in the operating room. Participants were monitored by pulse oximeter and noninvasive manometer. The participants were put in a supine position with an adjusted Fluorostar $7900 \mathrm{C}$-arm (GE Healthcare, Wendelstein, Germany) (Fig. 2A). After aseptic preparation, a 23-gauge spinal needle was placed in the middle third of the glenohumeral joint. Needle placement was confirmed with a glenohumeral arthrogram after injection of $1 \mathrm{~mL}$ of nonionic radiopaque contrast material (Fig. 2B). After confirmation, 2\% lidocaine $(5 \mathrm{~mL})$, contrast dye $(5 \mathrm{~mL})$, triamcinolone $(40 \mathrm{mg})$, and normal saline $(9 \mathrm{~mL})$, in a total fluid volume of $20 \mathrm{~mL}$, were injected under fluoroscopic imaging (Fig. 2C).

\section{Ultrasonography-guided capsular distension method} (group B)

The participants were put in a sitting position in a chair with the affected shoulder adducted and internally rotated and the elbow flexed to grasp the contralateral shoulder. After aseptic preparation, the joint injection was performed using a posterolateral approach with a 23-gauge needle under LOGIQ P5 ultrasound guidance (GE Healthcare) (Fig. 3A). After needle placement confirmation (Fig. 3B), 2\% lidocaine ( $5 \mathrm{~mL}$ ), triamcinolone (40 $\mathrm{mg}$ ), and normal saline (14 mL), for a total $20 \mathrm{~mL}$ fluid volume, were injected under sono-guidance (Fig. 3C).

\section{Physical therapy}

After injection, all patients received physiotherapy four times in the first week and then two times a week thereaf- 

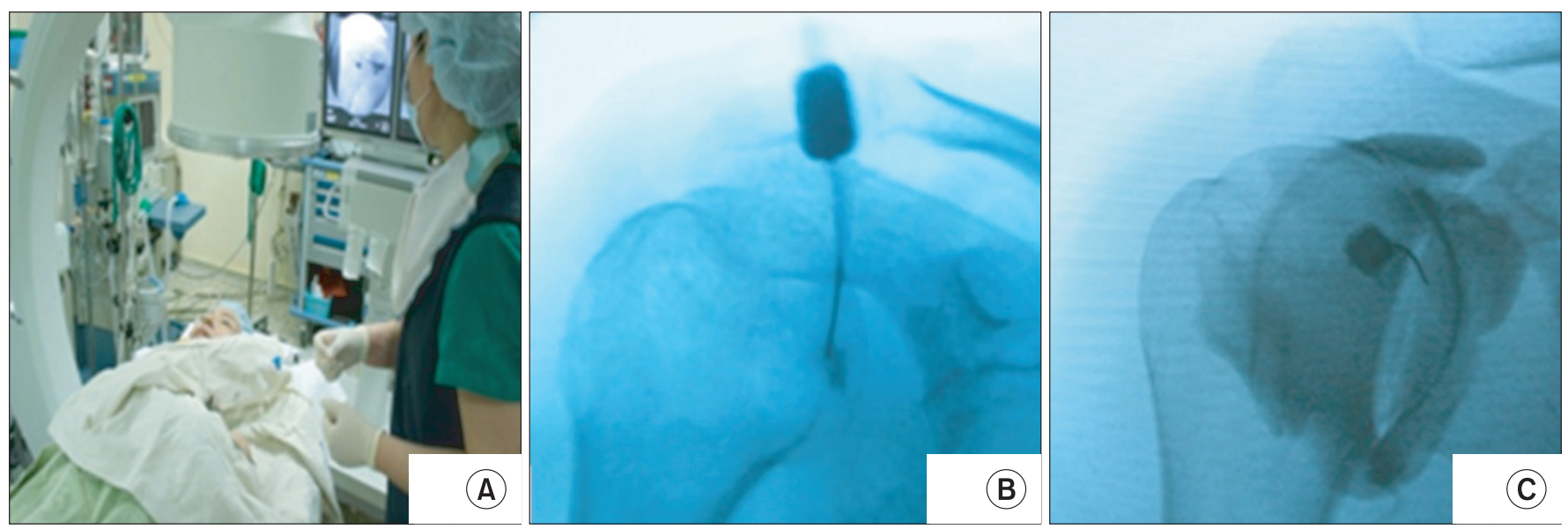

Fig. 2. Fluoroscopy-guided capsular distension method. (A) The participants were put in a supine position with a Carm adjusted in the operating room. (B) Needle placement was confirmed in a glenohumeral arthrogram after injection of $1 \mathrm{~mL}$ of nonionic radiopaque contrast material. (C) After confirmation, $2 \%$ lidocaine (5 mL), contrast dye (5 $\mathrm{mL}$ ), triamcinolone ( $40 \mathrm{mg}$ ), and normal saline $(9 \mathrm{~mL})$, for a total volume of $20 \mathrm{~mL}$, were injected under fluoroscopic imaging.
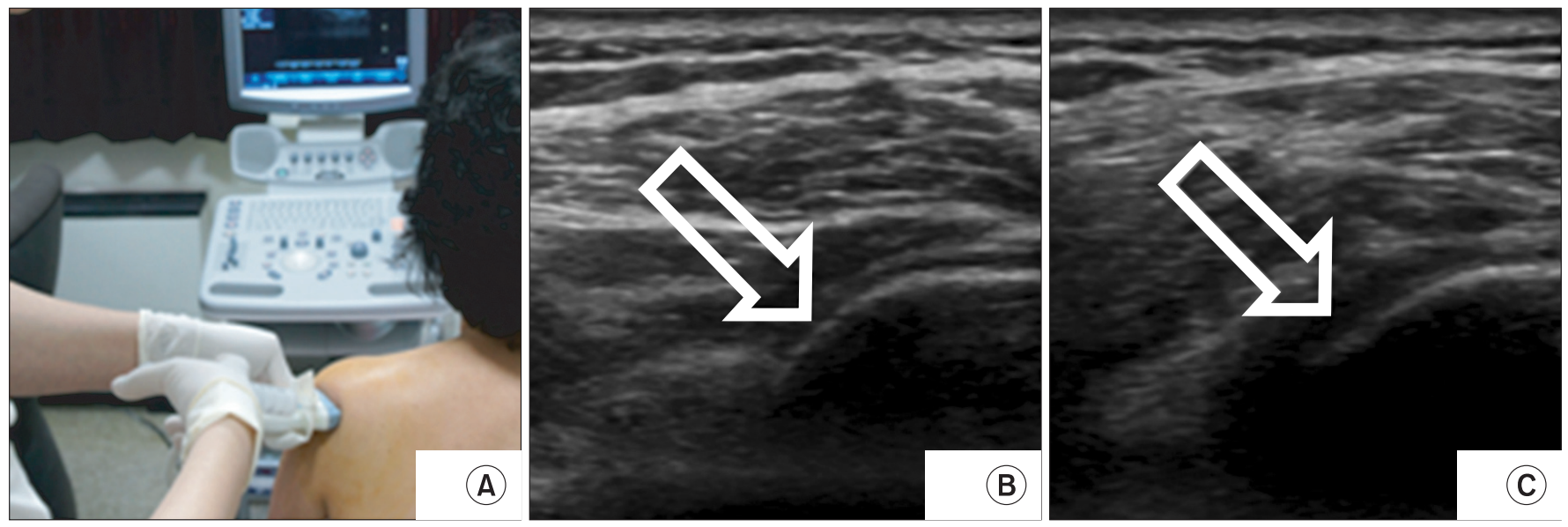

Fig. 3. Ultrasonography-guided capsular distension method. (A) The participants were put in a sitting position in a chair, and the affected shoulder was adducted and internally rotated and the elbow flexed to grasp the contralateral shoulder. (B) The joint injection was performed using a posterolateral approach with a 23-gauge needle under sonoguidance. (C) After needle placement confirmation, $2 \%$ lidocaine ( $5 \mathrm{~mL}$ ), triamcinolone ( $40 \mathrm{mg})$, and normal saline (14 $\mathrm{mL}$ ), for a total volume of $20 \mathrm{~mL}$ fluid, were injected under sono-guidance.

ter for eight weeks. The program was composed of physical modalities (heat and electrical therapy) and therapeutic exercise (gentle PROM exercise, active assisted ROM exercise, active ROM exercise, stretching exercise, and wand exercise, etc.).

\section{Outcome measures}

Treatment effects were assessed using the shoulder pain and disability index (SPADI), the visual numeric scale (VNS), passive range of motion (PROM) of the shoulder (flexion, abduction, and external rotation), hand grip, and pinch power at 4 times: baseline and at one week, five and nine weeks after injection.

\section{Shoulder pain and disability index}

This index is a measure of functional disability with a score from 0 (best) to 10 (worst) and was applied to eight categories in the present study: hair washing, washing 
of the back, putting on an undershirt or jumper, putting on a shirt that buttons down the front, putting on pants, placing an object on a high shelf, carrying a heavy 10 pound object, and removing something from a back pocket.

\section{Visual numeric scale}

The factor is a measure of shoulder pain, scored from 0 (no pain) to 10 (the worst pain imaginable).

\section{Passive range of motion of shoulder}

This variable measures shoulder motion ability with passive movement using a conventional goniometer. The measurement included abduction in the frontal plane, forward flexion, and external rotation with the arm at $0^{\circ}$ abduction.

\section{Hand grip and pinch power}

These variables are used to measure upper extremity power improvement. Grip strength was tested, followed by a key (lateral) pinch test. The participants held the Saehan Hydraulic Hand Dynamometer (Saehan, Masan, Korea) in the hand to be tested with their shoulder adducted and neutrally rotated, elbow flexed at $90^{\circ}$ and forearm in a neutral position. The handle of the dynamometer was adjusted until the base rested on the first metacarpal (heel of palm) and the handle rested in the middle of the four fingers. Participants squeezed the dynamometer with maximum isometric effort, which was maintained for about five seconds. No other body movement was allowed. The participants were strongly encouraged to give their maximum effort. The participants then held the pinch groove of JAMAR Hydraulic Pinch

Table 1. Characteristics of the participants

\begin{tabular}{lcc}
\hline \multicolumn{1}{c}{ Characteristic } & Group A (n=27) & Group B (n=27) \\
\hline Sex (female:male) & $22: 5(81.5: 18.5)$ & $17: 10(63.0: 37.0)$ \\
Age (yr) & $53.30 \pm 7.99$ & $53.85 \pm 6.61$ \\
$\begin{array}{l}\text { Symptom duration } \\
\text { (mo) }\end{array}$ & $7.22 \pm 4.69$ & $6.85 \pm 5.06$ \\
$\begin{array}{l}\text { Affected side } \\
\text { (right:left) }\end{array}$ & $16: 11(59.3: 40.7)$ & $13: 14(48.1: 51.9)$ \\
\hline
\end{tabular}

Values are presented as number (\%) or mean \pm standard deviation.

Group A, fluoroscopy-guided capsular distension; group $\mathrm{B}$, ultrasonography-guided capsular distension.
Gauge (Sammons Preston Rolyan, Nottinghamshire, UK) with the thumb pad and lateral aspect of the middle phalanx of the index finger. Participants pinched the gauge with maximum isometric effort. The pinch gauge was held at the distal end by the examiner to prevent dropping.

\section{Statistics}

Statistical data were analyzed with the SPSS software ver. 18 (SPSS Inc., Chicago, IL, USA). We investigated the difference in improvement of SPADI, VNS, PROM, and hand power between the two groups by a two-way repeated measures analysis of variance (ANOVA) with Bonferroni correction as the post-hoc test. The significance level was defined as a p-value less than 0.05 .

\section{RESULTS}

The general characteristics of 54 participants are shown in Table 1. The mean SPADI scores in groups A and $B$ were $37.37 \pm 17.36$ and $36.00 \pm 16.84$ (at baseline), $24.48 \pm 11.37$ and $18.41 \pm 13.51$ (at one week), 15.48 \pm 11.22 and $14.41 \pm 13.90$ (at five weeks), and $12.89 \pm 10.62$ and $12.93 \pm 13.75$ (at nine weeks), respectively. The SPADI scores improved one week after injection and continued to improve until nine weeks in both groups $(\mathrm{p}<0.05)$.

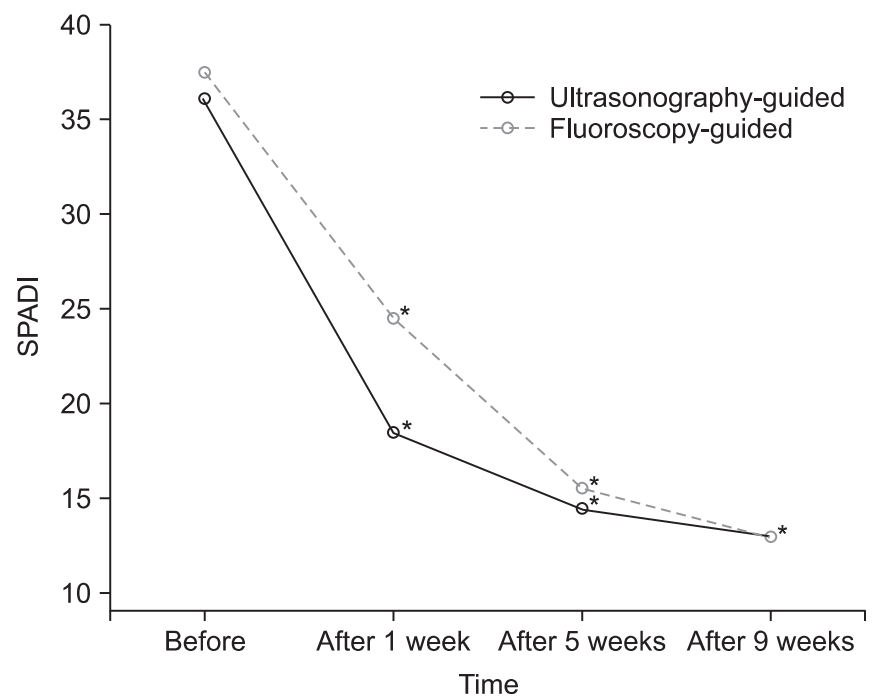

Fig. 4. Comparison of shoulder pain and disability index (SPADI) after capsular distension. SPADI was significantly improved in both groups $\left({ }^{*} \mathrm{p}<0.05\right)$. However, no statistically significant difference in the changes of SPADI was found between the two groups. 
However, no statistical differences in changes of SPADI scores between the two groups were identified $(p>0.05)$ (Fig. 4). The mean VNS scores in groups A and B were $6.48 \pm 18.82$ and $6.30 \pm 1.38$ (at baseline), $3.63 \pm 1.90$ and $2.81 \pm 1.81$ (at one week), $1.93 \pm 1.43$ and $2.15 \pm 1.89$ (at five weeks), and $1.48 \pm 1.28$ and $1.81 \pm 1.84$ (at nine weeks), respectively. The VNS scores improved one week after injection and continued to improve until nine weeks in the two groups $(\mathrm{p}<0.05)$. However, no statistical differences in changes of VNS scores between the two groups were seen ( $p>0.05$ ) (Fig. 5).

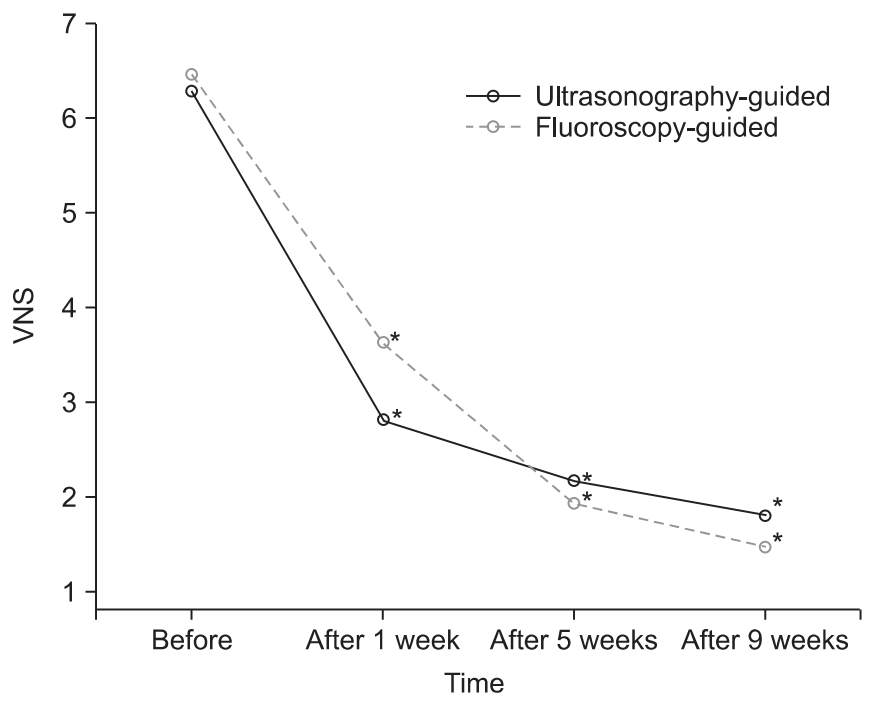

Fig. 5. Comparison of visual numeric scale (VNS) after capsular distension. VNS was significantly improved in groups A and B $\left({ }^{*} \mathrm{p}<0.05\right)$. However, no statistically significant difference in the VNS changes was found between the two groups.
PROM of the shoulder showed improvement one week after injection and continued to improve up until nine weeks in two groups $(\mathrm{p}<0.05)$. However, no statistical differences in changes of PROM were found between groups $A$ and $B$ (Table 2). The mean hand grip power scores in groups A and B were $21.59 \pm 11.60$ and $22.15 \pm 10.00$ $\mathrm{kg}$ (at baseline), $24.11 \pm 11.28$ and $24.57 \pm 8.98 \mathrm{~kg}$ (at one week), $24.61 \pm 10.68$ and $24.81 \pm 8.91 \mathrm{~kg}$ (at five weeks), and $25.13 \pm 11.11$ and $25.44 \pm 9.20 \mathrm{~kg}$ (at nine weeks), respectively. Hand grip power showed improvement one week after injection and continued to improve until nine weeks

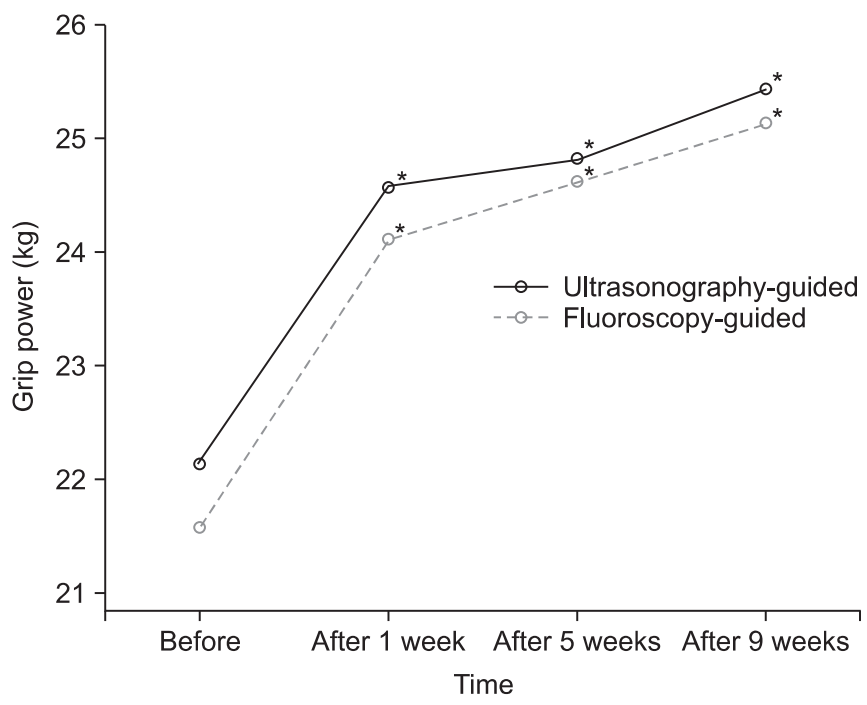

Fig. 6. Comparison of grip power after capsular distension. Grip power $(\mathrm{kg})$ was significantly improved in the two groups $\left({ }^{*} \mathrm{p}<0.05\right)$. However, no statistically significant difference in the changes of grip power was found between the two groups.

Table 2. Comparison of the PROM after capsular distension

\begin{tabular}{lccccc}
\hline \multirow{2}{*}{ Movement } & \multirow{2}{*}{ Group } & Before capsular & \multicolumn{3}{c}{ After capsular distension } \\
\cline { 4 - 6 } & & distension & $\mathbf{1 ~ w k}$ & $\mathbf{5}$ wk & $\mathbf{9}$ wk \\
\hline Flexion & $\mathrm{A}$ & $130.74 \pm 24.13$ & $152.41 \pm 23.39^{*}$ & $160.93 \pm 23.98^{*}$ & $162.59 \pm 23.22^{*}$ \\
\hline Abduction & $\mathrm{B}$ & $130.56 \pm 22.50$ & $153.89 \pm 19.43^{*}$ & $160.19 \pm 18.16^{*}$ & $163.15 \pm 16.30^{*}$ \\
\hline \multirow{2}{*}{ External rotation } & $\mathrm{A}$ & $109.63 \pm 26.71$ & $131.85 \pm 25.46^{*}$ & $144.81 \pm 28.44^{*}$ & $147.22 \pm 28.83^{*}$ \\
& $\mathrm{~B}$ & $104.81 \pm 21.90$ & $125.93 \pm 23.41^{*}$ & $134.81 \pm 23.76^{*}$ & $139.07 \pm 24.61^{*}$ \\
& $\mathrm{~A}$ & $40.74 \pm 22.90$ & $58.70 \pm 25.48^{*}$ & $68.15 \pm 23.54^{*}$ & $70.37 \pm 22.10^{*}$ \\
\hline
\end{tabular}

Values are presented as number (\%) or mean \pm standard deviation.

PROM, passive range of motion; group A, fluoroscopy-guided capsular distension; group B, ultrasonography-guided capsular distension.

${ }^{*} \mathrm{p}<0.05$, comparison of before vs. 1 week, 5 and 9 weeks after injection. 
in both groups $(\mathrm{p}<0.05)$. However, no statistical differences in changes of hand grip power were found between the two groups ( $p>0.05$ ) (Fig. 6). The mean hand pinch power in groups A and B were $6.81 \pm 2.60$ and $6.33 \pm 2.13 \mathrm{~kg}$ (at baseline), $7.48 \pm 2.49$ and $7.17 \pm 1.91 \mathrm{~kg}$ (at one week), $7.69 \pm 2.57$ and $7.28 \pm 1.91 \mathrm{~kg}$ (at five weeks), and $7.81 \pm 2.53$ and $7.37 \pm 1.95 \mathrm{~kg}$ (at nine weeks), respectively. Hand pinch power showed improvement one week after injection and continued to improve until nine weeks in the two groups $(\mathrm{p}<0.05)$. However, no statistical differences in changes of hand pinch power were found between groups A and B ( $p>0.05)$ (Fig. 7).

\section{DISCUSSION}

Adhesive capsulitis has been described as having three sequential phases: a painful stage, a freezing stage, and a thawing or recovery stage. However, there is no evidence to validate this classification, and its clinical utility is questionable. Pain and a limited ROM can occur in all phases of adhesive capsulitis, which often does not follow a stepwise course. In this condition, pain and decreased ROM can persist for one to two years, and up to $10 \%$ of patients never recover a full ROM [17]. It is important for physicians to decide which treatment is effective and timely as well as appropriate for patients with adhesive

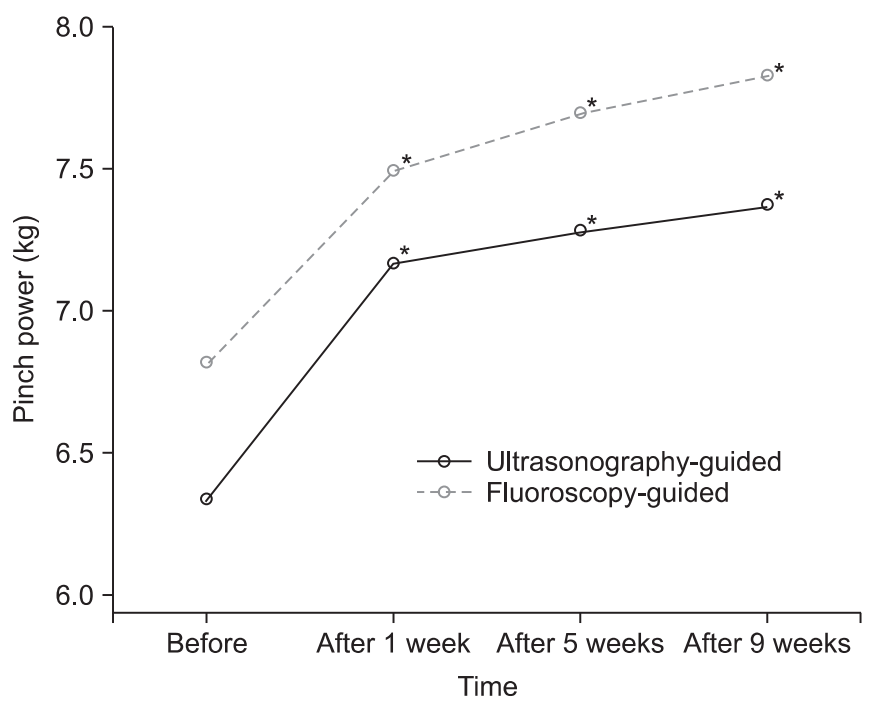

Fig. 7. Comparison of pinch power after capsular distension. Pinch power $(\mathrm{kg})$ was significantly improved in the two groups $\left({ }^{*} p<0.05\right)$. However, no statistically significant difference in changes of pinch power was found between the two groups. capsulitis.

Marx et al. [3] proposed that steroid injections provide chemical ablation of the synovitis, thus limiting the subsequent development of fibrosis and shortening the natural history of the disease. Tveita et al. [13] reported that the patients treated with distension plus steroids showed significant improvement in various ROM measures and experienced pain reduction compared with the group treated with steroids alone. Capsular distension is thought to exert its positive effects by improving glenohumeral mobility via stretching or rupturing of the joint capsule.

Park et al. [18] reported that ultrasonography-guided capsular distension using a posterolateral approach had comparable effects with fluoroscopy-guided capsular distension with a posterior approach for treatment of adhesive capsulitis of the shoulder and can be substituted for fluoroscopic capsular distension, which is advantageous from the viewpoint of radiation hazard mitigation, time, cost-effectiveness, and convenience. However, the rotator interval, a triangular anatomic area in the anterosuperior aspect of the shoulder, was taken into account as an important part of the pathology in adhesive capsulitis [19]. For this reason, we expected that an anterior approach to capsular distension would have better efficacy compared with a posterior approach. But the result of this study showed no difference between the treatments. Compared with capsular distension using a posterolateral approach, the anterior approach showed no better improvement of PROM. After capsular distension, the degree of improvement in the ROM is an important factor that can be used to evaluate the effectiveness of the treatment. Limitation of motion in patients with adhesive capsulitis is prominent in external rotation followed by abduction, internal rotation and flexion [20]. Levine et al. [21] reported that patients who were initially evaluated to have more limited ROM, especially for forward flexion and external rotation of their shoulders, were more likely to require surgical treatment. After capsular distension, Park and Hwang [22] found that forward flexion and internal rotation were improved significantly, while Choi et al. [23] reported that forward flexion, abduction and external rotation experienced the same positive results. Kim et al. [24] reported that forward flexion and abduction also significantly improved. We found that forward flexion, abduction and external rotation showed significant improvement in both treatment groups with no sta- 
tistically significant difference. Because internal rotation range was initially normal in the most participants, we didn't compare these results.

The positive results were attributed by the pathology of the anterior capsule of adhesive capsulitis. But the results of anterior approach study were no different than those of posterior approach.

Shoulder pain, range of movement, and disability have been the most commonly used endpoints in clinical trials for shoulder disorders. Chronic muscle pain is associated with abnormal patterns of muscular activity, resulting in altered movement strategies. Many patients with adhesive capsulitis complained of hand weakness. Dupeyron et al. [25] reported that pain, ROM, function, and strength are important components for evaluating shoulder disorders. Staples et al. [26] discovered that the SPADI and disabilities of the arm, shoulder and hand (DASH) have acceptable construct validity for patients with adhesive capsulitis. In this study, we measured eight disability categories of SPADI and hand grip and pinch power instead of using the DASH questionnaire for the evaluation of functional disability. There were no statistically significant differences between the treatment groups. We tried to know about how adhesive capsulitis affect upper extremity strength, it was different from other studies. A limitation of this study was that we used two different imaging guidance methods, even though they showed similar efficacy in previous studies.

We expected an anterior approach to capsular distension to be better than a posterolateral approach because the rotator interval was considered an important component of the pathology in adhesive capsulitis. However, ultrasonography-guided capsular distension by a posterolateral approach had outcomes similar to fluoroscopyguided capsular distension using an anterior approach.

\section{CONFLICT OF INTEREST}

No potential conflict of interest relevant to this article was reported.

\section{REFERENCES}

1. Neviaser AS, Neviaser RJ. Adhesive capsulitis of the shoulder. J Am Acad Orthop Surg 2011;19:536-42.

2. Rizk TE, Pinals RS. Frozen shoulder. Semin Arthritis Rheum 1982;11:440-52.
3. Marx RG, Malizia RW, Kenter K, Wickiewicz TL, Hannafin JA. Intra-articular corticosteroid injection for the treatment of idiopathic adhesive capsulitis of the shoulder. HSS J 2007;3:202-7.

4. Griesser MJ, Harris JD, Campbell JE, Jones GL. Adhesive capsulitis of the shoulder: a systematic review of the effectiveness of intra-articular corticosteroid injections. J Bone Joint Surg Am 2011;93:1727-33.

5. Huang CC, Tsao SL, Cheng CY, Hsin MT, Chen CM. Treating frozen shoulder with ultrasound-guided pulsed mode radiofrequency lesioning of the suprascapular nerve: two cases. Pain Med 2010;11:1837-40.

6. Chen HS, Chang KH, Kang JH, Hsu YH, Hsu AC, Chen SC. Treatment of frozen shoulder with intra-articular injection of botulinum toxin in shoulder joints. Med Hypotheses 2011;77:305-7.

7. Thomas WJ, Jenkins EF, Owen JM, Sangster MJ, Kirubanandan R, Beynon C, et al. Treatment of frozen shoulder by manipulation under anaesthetic and injection: does the timing of treatment affect the outcome? J Bone Joint Surg Br 2011;93:1377-81.

8. Andren L, Lundberg BJ. treatment of rigid shoulders by joint distension during arthrography. Acta Orthop Scand 1965;36:45-53.

9. Quraishi NA, Johnston P, Bayer J, Crowe M, Chakrabarti AJ. Thawing the frozen shoulder: a randomised trial comparing manipulation under anaesthesia with hydrodilatation. J Bone Joint Surg Br 2007;89:1197200.

10. Piotte F, Gravel D, Moffet H, Fliszar E, Roy A, Nadeau $\mathrm{S}$, et al. Effects of repeated distension arthrographies combined with a home exercise program among adults with idiopathic adhesive capsulitis of the shoulder. Am J Phys Med Rehabil 2004;83:537-46.

11. Park KD, Nam HS, Lee JK, Kim YJ, Park Y. Treatment effects of ultrasound-guided capsular distension with hyaluronic acid in adhesive capsulitis of the shoulder. Arch Phys Med Rehabil 2013;94:264-70.

12. Vad VB, Sakalkale D, Warren RF. The role of capsular distention in adhesive capsulitis. Arch Phys Med Rehabil 2003;84:1290-2.

13. Tveita EK, Tariq R, Sesseng S, Juel NG, Bautz-Holter E. Hydrodilatation, corticosteroids and adhesive capsulitis: a randomized controlled trial. BMC Musculoskelet Disord 2008;9:53.

14. Gondim Teixeira PA, Balaj C, Chanson A, Lecocq S, Louis M, Blum A. Adhesive capsulitis of the shoul- 
der: value of inferior glenohumeral ligament signal changes on T2-weighted fat-saturated images. AJR Am J Roentgenol 2012;198:W589-96.

15. Gaskill TR, Braun S, Millett PJ. The rotator interval: pathology and management. Arthroscopy 2011;27:55667.

16. Harryman DT 2nd, Sidles JA, Harris SL, Matsen FA $3 \mathrm{rd}$. The role of the rotator interval capsule in passive motion and stability of the shoulder. J Bone Joint Surg Am 1992;74:53-66.

17. Ewald A. Adhesive capsulitis: a review. Am Fam Physician 2011;83:417-22.

18. Park KD, Nam HS, Kim TK, Kang SH, Lim MH, Park Y. Comparison of sono-guided capsular distension with fluoroscopically capsular distension in adhesive capsulitis of shoulder. Ann Rehabil Med 2012;36:88-97.

19. Uhthoff HK, Boileau P. Primary frozen shoulder: global capsular stiffness versus localized contracture. Clin Orthop Relat Res 2007;456:79-84.

20. Park SK, Nam MH, Yune SH. Hydraulic management of frozen shoulder. J Korean Acad Rehabil Med 1992;16:147-53.

21. Levine WN, Kashyap CP, Bak SF, Ahmad CS, Blaine TA, Bigliani LU. Nonoperative management of id- iopathic adhesive capsulitis. J Shoulder Elbow Surg 2007;16:569-73.

22. Park GY, Hwang SE. Comparison of intraarticular steroid injection with and without capsular distension in adhesive capsulitis of the shoulder. J Korean Acad Rehabil Med 2000;24:1174-9.

23. Choi ES, Kim JS, Lee YS, Shin BS, Ko YJ, Park SH. Arthrographic findings of frozen shoulder and therapeutic effect of distension arthrography. J Korean Acad Rehabil Med 2001;25:278-83.

24. Kim JS, Kwon JY, Lee WI, Kim JM. The additional effect of tear after passive exercise during distension arthrography in patients with frozen shoulder. J Korean Acad Rehabil Med 2008;32:324-6.

25. Dupeyron A, Gelis A, Sablayrolles P, Bousquet PJ, Julia $M$, Herisson $C$, et al. Heterogeneous assessment of shoulder disorders: validation of the Standardized Index of Shoulder Function. J Rehabil Med 2010;42:96772.

26. Staples MP, Forbes A, Green S, Buchbinder R. Shoulder-specific disability measures showed acceptable construct validity and responsiveness. J Clin Epidemiol 2010;63:163-70. 